\title{
Next-generation sequencing in Brazilian MODY patients: a pilot study
}

Lílian Araújo Caetano*, Lucas Santos de Santana, Antônio Marcondes Lerário, Márcia Nery,

Alexander Augusto de Lima Jorge, Milena Gurgel Teles

From 20th Brazilian Diabetes Society Congress

Porto Alegre, Brazil. 11-18 November 2015

\section{Background}

Maturity-Onset Diabetes of the Young (MODY) is the most common form of monogenic diabetes. Genetic analysis is required to confirm the diagnosis. Conventional genetic testing uses Sanger sequencing. Currently, Next-generation sequencing (NGS) has proven to be cost-effective. To date, there is no NGS study for MODY in Brazil.

\section{Objective}

To validate a new assay for molecular diagnosis of MODY using targeted-NGS.

\begin{tabular}{|c|c|c|c|c|c|c|c|c|}
\hline Subject & $\begin{array}{l}\text { Age of } \\
\text { diabetes } \\
\text { onset } \\
\text { (years old) }\end{array}$ & $\begin{array}{l}\text { Family } \\
\text { history of } \\
\text { diabetes } \\
\text { (consecutive } \\
\text { generations) }\end{array}$ & Clinical Features & $\begin{array}{c}\text { Glucose } \\
\text { tolerance test }\end{array}$ & $\begin{array}{l}\text { Tests after at least } 3 \\
\text { years of diabetes } \\
\text { diagnosis }\end{array}$ & $\begin{array}{c}\text { Initial } \\
\text { Diagnostic } \\
\text { Hypothesis }\end{array}$ & Sanger sequencing & Next-generation sequencing \\
\hline 1 & 6 & 3 & $\begin{array}{l}\text { Low NPH insulin dose in the first } \\
\text { year of diagnosis. No diabetes } \\
\text { medication for } 3.5 \text { years, with } \\
\text { good givcemic control }\end{array}$ & $\begin{array}{c}\sigma^{\prime}: 138 \mathrm{mg} / \mathrm{dl} \\
12 \sigma^{\prime}: 145 \mathrm{mg} / \mathrm{dt}\end{array}$ & $\begin{array}{l}\text { FPG } 125 \mathrm{mg} / \mathrm{dl} \\
\text { Alc } 6.7 \% \\
\text { FCP } 1.1 \mathrm{ng} / \mathrm{mL}\end{array}$ & $\begin{array}{l}\text { MOOY2 } \\
(G C K)\end{array}$ & $\begin{array}{c}G \alpha^{\prime} \\
\text { C.580-3C>A(INSS) }\end{array}$ & $\begin{array}{c}G \alpha^{\prime} \\
\text { C.580-3CA(IVSS) }\end{array}$ \\
\hline 2 & 4 & 3 & $\begin{array}{l}\text { Mild hyperglycemia with } \\
\text { worsening only under stress } \\
\text { conditions (eg: infection). } \\
\text { No diabetes medication for } 38 \\
\text { years and good glycemic control }\end{array}$ & NA & $\begin{array}{l}\text { FPG } 136 \mathrm{mg} / \mathrm{dl} \\
\text { Alc } 6.5 \% \\
\text { FCP } 1.6 \mathrm{ng} / \mathrm{mL}\end{array}$ & $\begin{array}{l}\text { MOOY2 } \\
(G C K)\end{array}$ & $\begin{array}{c}G \alpha^{\prime} \\
\text { c.SOSA } \sigma / p . K 169 E\end{array}$ & $\begin{array}{c}G Q^{\prime} \\
c .505 A>G / p . K 169 E\end{array}$ \\
\hline 3 & 14 & 2 & $\begin{array}{l}\text { Excellent glycemic control with a } \\
\text { low dose sulfonylurea }\end{array}$ & $\begin{array}{c}\sigma^{\prime}: 98 \mathrm{mg} / \mathrm{dl} \\
120^{\circ}: 214 \mathrm{mg} / \mathrm{dl}\end{array}$ & $\begin{array}{l}\text { FPG } 112 \mathrm{mg} / \mathrm{dl} \\
\text { A1c S.3\% } \\
\text { FCP } 2.2 \mathrm{ng} / \mathrm{mL}\end{array}$ & $\begin{array}{l}\text { MODY3 } \\
\text { (HNF1A) }\end{array}$ & $\begin{array}{c}\text { HNF1A } \\
\text { c.15580T/p.0520 }\end{array}$ & $\begin{array}{c}\text { HNF } 1 A^{2} \\
\text { c.15580T/p.0520. }\end{array}$ \\
\hline 4 & 35 & 2 & $\begin{array}{l}\text { Good glycemic control with no } \\
\text { diabetes medication. Insulin } \\
\text { required only during pregnancy }\end{array}$ & $\begin{array}{c}\sigma^{\prime}: 108 \mathrm{mg} / \mathrm{dt} \\
12 \sigma^{\circ}: 197 \mathrm{mg} / \mathrm{dt}\end{array}$ & $\begin{array}{l}\text { FPG } 120 \mathrm{mg} / \mathrm{dl} \\
\text { A1c } 5.2 \% \\
\text { FCP } 2.3 \mathrm{ng} / \mathrm{mL}\end{array}$ & $\begin{array}{l}\text { MOOY2 } \\
(G C K)\end{array}$ & Not performed & $\begin{array}{c}G C K \\
c .952 G>N / D . G 318 R\end{array}$ \\
\hline 5 & 30 & 3 & Poor treatment compliance & NA & $\begin{array}{l}\text { FPG } 315 \mathrm{mg} / \mathrm{dl} \\
\text { A1c } 10.3 \% \\
\text { FCP } 1.3 \mathrm{ng} / \mathrm{mL}\end{array}$ & $\begin{array}{l}\text { MODY3 } \\
\text { (HNF1A) }\end{array}$ & Not performed & $\begin{array}{c}\text { HNF1A } \\
\text { c.1781G>T/p.SS94I }\end{array}$ \\
\hline 6 & 15 & 4 & $\begin{array}{c}\text { Asymptomatic hyperglycemia. } \\
\text { Good control with no diabetes } \\
\text { medication }\end{array}$ & $\begin{array}{c}\sigma^{\gamma}: 111 \mathrm{mg} / \mathrm{dl} \\
12 \sigma^{\circ}: 182 \mathrm{mg} / \mathrm{dl}\end{array}$ & $\begin{array}{l}\text { FPG } 122 \mathrm{mg} / \mathrm{dl} \\
\text { Alc } 6.2 \% \\
\text { FCP } 1.6 \mathrm{ng} / \mathrm{mL}\end{array}$ & $\begin{array}{l}\text { MOOY2 } \\
(G C K)\end{array}$ & GCK-Negative & $\begin{array}{c}G C K \\
\text { C.1340_1368del29/p.R447tss*2 }\end{array}$ \\
\hline 7 & 8 & 2 & $\begin{array}{l}\text { Beginning sulfonylurea } 3 \text { years } \\
\text { after diabetes diagnosis. On } \\
\text { treatment, A1c levels around } 7 \%\end{array}$ & $\begin{array}{c}\sigma^{\prime}: 114 \mathrm{mg} / \mathrm{dl} \\
12 \sigma^{\circ}: 106 \mathrm{mg} / \mathrm{dt}\end{array}$ & $\begin{array}{l}\text { FPG } 125 \mathrm{mg} / \mathrm{dl} \\
\text { Alc } 7.2 \% \\
\text { FCP } 2.8 \mathrm{ng} / \mathrm{mL}\end{array}$ & $\begin{array}{l}\text { MOOY3 } \\
\text { (HNF1A) }\end{array}$ & HNF1A-Negative & $\begin{array}{c}G C K \\
c .544 G>A / p . V 182 \mathrm{M}\end{array}$ \\
\hline
\end{tabular}

All patients have negative pancreatic antibodies (GAD, IA2, IMA).

FPG, fasting plasma glucose. A1c, glycated hemoglobin. FCP, fasting C-peptide. NA, not available.

'Novel mutations, not previously described in the literature.

Figure 1 Mutations identified by NGS in Brazilian subjects with MODY phenotype.

\footnotetext{
* Correspondence: liliancaetano@yahoo.com.br

USP, São Paulo, Brazil 


\section{Materials and methods}

We have completed a pilot project including 7 unrelated subjects with MODY phenotype. Genetic sequencing was performed using Illumina NGS platform (MiSeq), allowing analysis of 13 MODY genes simultaneously. All exonic and intronic regions of these genes were evaluated. Two cases were not tested before. In other 5 cases, a previous analysis using Sanger sequencing of GCK and HNF1A genes had been done. Three subjects had already a genetic diagnosis of MODY by Sanger method and were selected to validate NGS Results. And the other 2 cases had typical clinical features but negative Sanger analysis.

\section{Results}

In all 7 cases analyzed, NGS was able to detect the mutations related to MODY, and in those 2 previous negative Sanger sequencing, it has allowed us to confirm the diagnosis of this type of diabetes (Figure 1). Considering these 2 negative Sanger cases, one had a mild hyperglycemia detected at 15 yo, non-progressive during 4 yrs. of follow-up, normal C-peptide, negative beta cell antibodies, and also a family history of similar phenotype. Previous Sanger testing for GCK had yielded a false negative result, because the mutation was a large deletion located at the end of the last exon of GCK, which impaired the analysis by Sanger, however was detected by NGS. The second negative Sanger subject had diabetes since 8 yo, low BMI, negative antibodies, detectable fasting C-peptide, and a mother with asymptomatic hyperglycemia. This patient had been using sulfonylurea with good glycemic control. Sanger sequencing for HNF1A was negative. NGS identified a mutation in GCK, already described in the literature as pathogenic.

\section{Conclusions}

In our pilot project, targeted-NGS was able to confirm MODY diagnosis in all cases submitted to Sanger sequencing ( 3 positive controls and 2 previously negative cases). In other two not tested before, this new method could identify the pathogenic variants. Thus, NGS can be considered an effective tool for diagnosing clinical suspicious cases of MODY, appearing to be a promising technique.

Published: 11 November 2015

doi:10.1186/1758-5996-7-S1-A257

Cite this article as: Caetano et al:: Next-generation sequencing in

Brazilian MODY patients: a pilot study. Diabetology \& Metabolic Syndrome 2015 7(Suppl 1):A257.
Submit your next manuscript to BioMed Central and take full advantage of:

- Convenient online submission

- Thorough peer review

- No space constraints or color figure charges

- Immediate publication on acceptance

- Inclusion in PubMed, CAS, Scopus and Google Scholar

- Research which is freely available for redistribution

Submit your manuscript at www.biomedcentral.com/submit 